\title{
Predicting Perinatal Outcome Using Doppler in IUGR and PIH - Evaluating Cerebro Umbilical Ratio
}

\author{
First Author : Dr. K. Suriya Praba ${ }^{1}$ \\ Corresponding Author: Dr A. Shantha Vibala ${ }^{2}$ \\ ${ }^{I} M D(O \& G)$, Assistant Professor, Dept of Obstetrics \& Gynaecology, \\ Melmaruvathur Adhiparasakthi Institute of Medical Sciences and Research \\ ${ }^{2}$ Senior Assistant Professor, Dept of Obstetrics \& Gynaecology, Govt Theni Medical College, Theni
}

\section{Introduction}

IUGR is said to be present in those babies whose birth weight is below the tenth percentile of the average for the gestational age. Incidence among term babies is 5\%.Incidence in preterm babies is $15 \%$. Lubchenaco and co-workers published norms for expected fetal weight at a given gestational week, Battalgia and Lubchenaco then classified small for gestational age infants as those whose Birth weight were below the 10th percentile for their gestetional age. These infants were shown to be at increased risk for Neonatal death. Neonatal mortality rate of SGA infant born at 38 wks was $1 \%$ compared with that of $0.2 \%$ in those with appropriate birth weight. There are several nomogroms to determine percentile distribution of birth weights at different gestational ages. Nomograms by Brenner et. al., (1976), Alexandar et. al., (1996), Arbuckle et. al., (1993), and finally Meintire et. al., (1999) are in use. Many SGA fetuses are not pathologically growth restricted but are small, normal biological fetuses. Maning and Holder 1991, Gordosi and Colleques (1992) proposed 25 percent to 60 percent of SGA infants were appropriately grown when maternal ethnic group, parity, weight and height were considered. Because of these disparities seeds, in 1984 suggested definition based on birth weight below the fifth percentile. Usher and Mclean (1969) proposed that Fetal growth standards should be based on mean weight for Age with normal limits defined by 2 standard deviation. As per this, SGA infants limits to 3 percent instead of 10 percent.

To evaluate the screening efficiency of

\section{Aim Of The Study}

1. Ratio of Pulsatility indicies of umblical and the middle cerebral arteries.

2. The pulsatility index of the umblical artery.

3. The pulsatility index of the middle cerebral artery in predicting the adverse perinatal outcome in pregnancies complicated by intrauterine growth restriction.

\section{Materials And Methods}

This study was conducted in the Obstetrics and Gynaecology department, Theni Medical College and Hospital, between March 2013- October 2014. A total of 90 singleton pregnancies constituted the study population.

\section{Inclusion criteria:}

1. Women who had regular menstrual cycles prior to conception and sure of their last menstrual period (LMP) or whose gestational age had been established by first trimester ultrasonogram.

2. Women with singleton pregnancy.

3. Women of gestational age between 30 and 40 weeks of pregnancy.

4. Women identified to have IUGR and women with high-risk pregnancies like pregnancy induced hypertension, hypertension complicating pregnancy and other conditions with associated fetal growth restriction.

5. Pregnancies beyond 30 weeks of gestation complicated by severe pre eclampsia and intra uterine growth restriction (or) both were selected.

Gestational age determination was based on a best estimate from menstrual historal, clinical gestational age and fetal biometry. Severe preecclampsia defined according to the standard criteria of uncontrolled blood pressure, proteinuria and ultrasound parameters of IUGR. Other pregnancy complications like gestational diabetes are also included if associated with severe PIH. 


\section{Exclusion criteria:}

1. Women with fetuses with major congenital malformation diagnosed by a prenatal ultrasonogram.

\section{Method:}

Doppler examinations were performed with a LOGIQ 500 duplex doppler scanner (GE medical systems) using a $2.5 \mathrm{MHz}$ tranducer. Studies were done with the patient in semi-recumbent position and the fetus in a quiet resting and apnoeic state. This was determined both by direct observation using real-time ultrasound and by the absence of doppler waveform patterns typical of gross fetal movements and breathing, i.e., irregularity of arterial waveforms and swaying of the venous waveforms. The Doppler determinations were done by one consultants in the unit for all the study patients.

\section{Results}

Age Distribution

\begin{tabular}{|c|c|c|}
\hline \multirow{2}{*}{ Age group } & \multicolumn{2}{|c|}{ cases } \\
\cline { 2 - 3 } & 5 & 5.6 \\
\hline Less than 20 yrs & 29 & 32.2 \\
\hline $20-24$ & 40 & 44.4 \\
\hline $25-29$ & 16 & 17.8 \\
\hline $30 \&$ above & 90 & 100 \\
\hline Total & $18-40$ yrs & \\
\hline Range & 26 & \\
Mean & 4.4 yrs & \\
S.D & & \\
\hline
\end{tabular}

\section{Obstetric Code}

\begin{tabular}{|c|c|c|}
\hline \multirow{2}{*}{ OBS.CODE } & \multicolumn{2}{|c|}{ CASES } \\
\cline { 2 - 3 } & NO. & 65.6 \\
\hline PRIMI & 59 & 26.7 \\
\hline MULTI & 24 & 4.4 \\
\hline G2 & 4 & 1.1 \\
\hline G4 & 1 & 2.2 \\
\hline G5\& ABOVE & 2 & 34.4 \\
\hline MULTI & 31 & 100 \\
\hline
\end{tabular}

Gestational Age

\begin{tabular}{|c|c|c|}
\hline \multirow{2}{*}{ GESTATIONAL AGE } & \multicolumn{2}{|c|}{ CASES } \\
\cline { 2 - 3 } & NO. & $\%$ \\
\hline PRETERM & 41 & 45.6 \\
\hline TERM & 49 & 54.4 \\
\hline TOTAL & 90 & 100 \\
\hline
\end{tabular}

\begin{tabular}{|c|c|c|c|}
\hline \multicolumn{5}{|c|}{ Weight Of Mother/Baby (Kgs) } \\
\hline WEIGHT & RANGE & MEAN & S.D \\
\hline MOTHER & $42-68$ & 52.3 & 6 \\
\hline BABY & $1-4.5$ & 2.13 & 0.69 \\
\hline
\end{tabular}

Antenatal Risk Factor

\begin{tabular}{|c|c|c|}
\hline \multirow{2}{*}{ ANTENATAL RISK FACTOR } & NO. & \multicolumn{2}{c|}{ CASES } \\
\cline { 2 - 4 } & $\mathbf{6 8}$ & $\mathbf{7 4 . 4}$ \\
\hline PIH & 6 & $\mathbf{6 . 7}$ \\
\hline PEVERE PIH & 1 & 1.1 \\
\hline IUGR & 10 & 11.1 \\
\hline BOH & 2 & 2.2 \\
\hline NIL & 1 & 1.1 \\
\hline TOTAL & 2 & 2.2 \\
\hline
\end{tabular}

Umblical Artery- P.I

\begin{tabular}{|c|c|c|}
\hline \multirow[t]{2}{*}{ UMBLICAL ARTERY P.I } & \multicolumn{2}{|c|}{ CASES } \\
\hline & NO. & $\%$ \\
\hline NORMAL $(<1.12)$ & 48 & 53.3 \\
\hline ABNORMAL(>/=1.12) & 42 & 46.7 \\
\hline TOTAL & 90 & 100 \\
\hline $\begin{array}{l}\text { RANGE } \\
\text { MEAN }\end{array}$ & \multicolumn{2}{|c|}{$\begin{array}{c}0.82-1.8 \\
1.13\end{array}$} \\
\hline
\end{tabular}


Predicting Perinatal Outcome Using Doppler In IUGR and PIH - Evaluating Cerebro Umbilical Ratio \begin{tabular}{l|l} 
S.D & 0.18 \\
\hline
\end{tabular}

Middle Cerebral Artery -P.I

\begin{tabular}{|c|c|c|}
\hline \multirow{2}{*}{ MIDDLE CEREBRAL ARTERY P.I } & \multicolumn{2}{|c|}{ CASES } \\
\hline & NO. & $\%$ \\
\hline NORMAL(>1.12) & 51 & 56.7 \\
\hline ABNORMAL $(</=1.12)$ & 39 & 43.3 \\
\hline TOTAL & 90 & 100 \\
\hline MCA/UA RATIO & & \\
\hline NORMAL $(>1.08)$ & 16 & 17.8 \\
\hline ABNORMAL $(<1.08)$ & 74 & 82.2 \\
\hline TOTAL & 90 & 100 \\
\hline
\end{tabular}

Range 0.6-1.38

Mean 1.03

Sd .13

\begin{tabular}{|c|l|c|}
\multicolumn{1}{|c}{ Types Of Labour } \\
\hline Type Of Labour & \multicolumn{1}{c|}{ No. } & $\%$ \\
\hline Spontaneous & \multicolumn{1}{c|}{4} & 4.4 \\
\hline Induction Of Labour & $\mathbf{3 1}$ & $\mathbf{3 4 . 4}$ \\
Induced Labour-Pih & $\mathbf{8}$ & $\mathbf{8 . 9}$ \\
Operative & 47 & 52.2 \\
Total & 90 & 100 \\
\hline
\end{tabular}

\begin{tabular}{|c|c|c|}
\hline \multicolumn{2}{|c|}{ Mode Of Delivery } \\
\hline Code Of Delivery & No. & \% \\
\cline { 2 - 3 } & 11 & 28.9 \\
\hline Ln With Epi & 26 & 1.1 \\
\hline Assisted Breech & 1 & 1.1 \\
\hline Outlet Forceps & 1 & 1.1 \\
\hline Lscs & & 44.4 \\
\hline Elective & 1 & 11.1 \\
Rpt & 40 & 56.7 \\
\hline Total Lscs & 10 & 100 \\
\hline
\end{tabular}

\section{Liquor}

\begin{tabular}{|c|c|c|}
\hline Liquor & No. & $\%$ \\
\hline Clear & 71 & 78.9 \\
\hline Oligo & 6 & 6.7 \\
\hline Msaf & 13 & 14.4 \\
\hline Total & 90 & 100 \\
\hline
\end{tabular}

Apgar Score At 5 Min

\begin{tabular}{|c|c|c|}
\hline \multirow{2}{*}{ Apgar Score At 5 Min } & \multicolumn{2}{|c|}{ Cases } \\
\cline { 2 - 4 } & No. & 35.6 \\
\hline$<7$ & 32 & $\mathbf{6 4 . 4}$ \\
\hline$>7$ & 58 & 100 \\
\hline
\end{tabular}

\begin{tabular}{|c|c|c|}
\hline \multicolumn{2}{|c|}{ Ceonatal Complications } \\
\hline Neonatal Complications & No. & $\%$ \\
\cline { 2 - 3 } & 23 & 5.6 \\
\hline Increased Br & 6 & 6.7 \\
\hline Hypoglycemia & 6 & 6.7 \\
\hline Hie & 2 & 2.2 \\
\hline Hypothermia & 1 & 1.1 \\
\hline Rds & 1 & 1.1 \\
\hline Sepsis & 1 & 1.1 \\
\hline Iud & 1 & 11.1 \\
\hline Neonatal Death & 10 & 3.3 \\
\hline Still Birth & 3 & 54.4 \\
\hline
\end{tabular}


Predicting Perinatal Outcome Using Doppler In IUGR and PIH - Evaluating Cerebro Umbilical Ratio

\begin{tabular}{|c|c|c|}
\hline Apgar <7 At 5 Min & 32 & 35.6 \\
\hline No Complication & 19 & 21.1 \\
\hline \multicolumn{3}{|c|}{ Perinatal Outcome } \\
\hline \multirow[t]{2}{*}{ Perinatal Outcome } & \multicolumn{2}{|c|}{ Cases } \\
\hline & No. & $\%$ \\
\hline Good(Alive \& No Complication) & 19 & 21.1 \\
\hline $\begin{array}{l}\text { Adverse(Death,Nicu } \\
\text { Admission) }\end{array}$ & 78.9 & \\
\hline Total & 100 & \\
\hline
\end{tabular}

\begin{tabular}{|c|c|c|c|c|c|c|c|}
\hline \multicolumn{8}{|c|}{ Relationship Between Cerebral Umbilical Ratio And Other Variables } \\
\hline \multirow[t]{3}{*}{ Variable } & \multicolumn{6}{|c|}{ Cerebral Umblical Ratio } & \multirow{3}{*}{$\mathbf{P}$} \\
\hline & \multicolumn{2}{|c|}{ Normal(1.08) } & \multicolumn{2}{|c|}{ Abnormal(<1.08) } & \multirow{2}{*}{$\begin{array}{l}\text { Me } \\
\text { an }\end{array}$} & \multirow[t]{2}{*}{ S.D } & \\
\hline & No. & $\%$ & No. & $\%$ & & & \\
\hline Age $<30(74)$ & 13 & 17.6 & 61 & 82.4 & 1 & 0.14 & 0.837 N0t S \\
\hline$>30(16)$ & 3 & 18.8 & 13 & 81.3 & 3 & 0.11 & Significant \\
\hline Weight & & & & & & & \\
\hline$<45 \operatorname{kg}(17)$ & 4 & 23.5 & 13 & 76.5 & $\mathbf{1}$ & 0.11 & $0-5603$ \\
\hline$>45 \mathrm{Kg}(73)$ & 12 & 16.4 & 61 & 83.6 & 4 & 0.14 & O.6598 \\
\hline Primi(59) & 11 & 18.6 & 48 & 81.4 & $\mathbf{1}$ & 0.15 & Notsignifica \\
\hline Multi(31) & 5 & 16.1 & 26 & 83.9 & 3 & 0.1 & nt \\
\hline \multirow{3}{*}{\multicolumn{2}{|c|}{ Antenatal Risk Factor }} & \multicolumn{6}{|c|}{ Cerebral Umbilical Ratio) } \\
\hline & & \multicolumn{3}{|c|}{ Normal 16 Cases (>1.08) } & \multicolumn{3}{|c|}{ Abnormal 74 Cases $(<1.08)$} \\
\hline & & \multicolumn{2}{|c|}{ No } & $\%$ & \multicolumn{2}{|c|}{ No } & $\%$ \\
\hline \multicolumn{2}{|c|}{$\operatorname{Pih}(67)$} & \multicolumn{2}{|c|}{12} & 17.9 & \multicolumn{2}{|c|}{55} & 82.1 \\
\hline \multicolumn{2}{|c|}{ Severe Pih(6) } & \multicolumn{2}{|l|}{-} & - & \multicolumn{2}{|c|}{6} & 100 \\
\hline \multicolumn{2}{|c|}{ Iugr(10) } & \multicolumn{2}{|l|}{2} & 20 & \multicolumn{2}{|c|}{8} & 80 \\
\hline \multicolumn{2}{|c|}{ Chronic Ht(2) } & \multirow{2}{*}{\multicolumn{2}{|c|}{-}} & - & \multicolumn{2}{|c|}{2} & 100 \\
\hline \multicolumn{2}{|c|}{ Other Medical Disorders(3) } & & & 33.3 & & & 66.7 \\
\hline
\end{tabular}

\begin{tabular}{|c|c|c|c|c|}
\hline \multirow{2}{*}{ Onset Of Labour } & \multicolumn{4}{|c|}{ Cerebral Umbilical Ratio } \\
\cline { 2 - 5 } & Normal 16 Cases & \multicolumn{2}{c|}{ Abnormal 74 Cases } \\
\cline { 2 - 5 } & No. & $\%$ & No & - \\
\hline Spontaneous Labour -4 & 4 & 100 & - & $\mathbf{8 2 . 1}$ \\
\hline Induction Of Labour-36 & 5 & 17.9 & 31 & $\mathbf{9 0}$ \\
\hline Emergency Lscs-40 & 4 & - & 1 & $\mathbf{1 0 0}$ \\
\hline Elective Lscs -1 & - & $\mathbf{3 0}$ & $\mathbf{7}$ & $\mathbf{7 0}$ \\
\hline Repeat Lscs-10 & $\mathbf{3}$ & & & \\
\hline
\end{tabular}

\section{Discussion}

The development of a good utero - placental circulation is essential for achievement of a normal pregnancy. IUGR is associated with an increased risk of perinatal mortality, morbidity and impaired neurodevelopment. The correct detection of the compromised IUGR fetus to allow for timely intervention is a main objective of antenatal care.

Cerebral - umbilical ratio is the ratio between the pulsatility indices of the middle cerebral artery and the ubilical artery. Previous studies done by Gramellini et. al., Arias et. al., and Singh el. al., had concluded that the cerebral umbilical was superior than the individual doppler indices of umbilical artery and middle cerebral arteries in predicting adverse perinatal outcome in IUGR fetuses. This study was done to evaluate the efficacy of the cerebral-umbilical ratio and pulsatility indices of the middle cerebral and umbilical arteries to predict adverse perinatal outcome in IUGR fetuses. From March 2013to October 2014, doppler indices namely the pulsatility indices of the umbilical artery and the middle cerebral artery and the cerebro umbilical ratio was studied in 90 (Ninety) women. Clinically suspected to have IUGR and in women with P1H. Doppler determination was performed with the patient in semi-recumbent position and the fetus in a quiet resting and apnoeic state. This was done between 30 - 40 weeks of pregnancy. The pulsatality indices of the umbilical artery, middle cerebral artery and the raio were calculated. The last value obtained before delivery was taken for the study. The doppler studies were done by one consultant for all the study patients. After a technically sstisfactory doppler waveform was obtained on the screen, the image was frozen. Pulsatility indices of the best three cardiac cycles were calculated and the average of them was obtained. The results of the doppler values were not revealed the consultant managing the patient. The patients were followed at the time of delivery and the perinatal outcome data obtained. Wladimiroff et. al., had shown that there was no significant variation in the cerebro umbilical ratio between 30 - 40 weeks of pregnancy. Gramellini et. al., studied twenty five women with normal pregnancies and obtained cutoff value of 1.08 for cerebro umbilical ratio. This was -2 standard 
deviations of the mean of the value obtained between $30-41$ weeks of gestations. A value $\leq 1.08$ was considered abnormal and value $>1.08$ was considered normal.. The same cerebral-umbilical ratio cutoff value of 1.08 was used in this study. The women were divided into 2 groups based on this cutoff value. The adverse perinatal outcome like low birth weight, low apgar scores, rate of cesarean section for fetal distress, meconium staining of the amniotic fluid, number of days of nursery care and the neonatal complications were analysed in both the groups. In this study the demographic variables like Age, height, weight of the women were similar in both the groups. In the abnormal ratio groups the percentage of women belonging to lower socio-economic status was found to be significally higher than in the normal ratio group $(92.5 \%$ vs $29 \%)$ with the 'p' value of 0.023. This indicates the incidence of SGA babies with abnormal cerebral-umbilical ratio is likely to be high in the women of lower socio-economic status category.

As similar finding had not been reported by others. The Antenatal risk factors that are associated with IUGR are studied in both groups. The percentages of women with an risk factors like pregnancy induced hypertension, hypertension complicating pregnancy, vascular disorders, maternal infection, and delivery of small for gestational age babies in the previous pregnancy were found to be higher in the abnormal ratio group compared to that in the normal ratio group through it was not statistically significant. Arias et. al., studied the resistance index in 61 antenatal women with similar risk factors and found that 22 women had abnormal cerebro-umbilical ratio. In women with clinically detected IUGR when ratio was $>1.08$ significant no. Of women were found to have no antenatal risk factor compared to women with ratio $\leq 1.08$ and the ' $\mathrm{p}$ ' value was 0.002 , C.I. $-0.03-0.51$. This indicates that when there is IUGR associated with antenatal risk factors. There is increased chance of having abnormal cerebro-umbilical ratio. The gestational age at delivery in the abnormal ratio group was significantly lower, than normal ratio group. The babies with IUGR and abnormal ratio were delivered much earlier, because of evidence of fetal distress.

There was more proportion of spontaneous labour when compared to induced labour in normal ratiogroup. Labour was induced in $82.1 \%$ abnormal ratio group. About 44 operative deliveries were performed in abnormal ratio group. The indication's for operative delivery was fetal distress and oligohydramnior in about 22 cases. In the rest of the cases it was due to severe PIH, induction failure (or) CPD. There was more number of labour natural and labour natural with episiotomy in the abnormal ratio group. This is due to more number of cases in abnormal ratio group than normal ratio group. More over about $78.4 \%$ of operative delivery occurred in abnormal ratio group. In the study by Grammelini et al., the rate of C.S was $88.8 \%$. In abnormal ratio group and $12.5 \%$ in the other group. In another study by arias et al. The rate of C.S. was $86.3 \%$ in the abnormal ratio group and $51.2 \%$ in normal ratio group. In this study the incidence of LSCS was $78.4 \%$ compared to $21.6 \%$ in normal ratio group. About $80 \%$ of neonatal deaths occurred in the abnormal ratio group. All the neonatal deaths in the abnormal group were due to IUGR complications. Among 2 neonatal death in normal ratio group, 1 case was extreme prematurity and the other was deeply asphyxiated baby. Meconium staining of the amniotic fluid was noted in the abnormal ratio group. In study by Singh et. al., did not find an increase in the incidence of meconium staining of amniotic fluid. The mean birth weight in abnormal ratio group was $2.15 \mathrm{~kg}$ where as it was $2.11 \mathrm{~kg}$ in the normal ratio group. The mean birth weight was higher in the abnormal ratio groups then the normal group in this study. This is because of more number of cases in the abnormal ratio group. But in the study by Grammelini et al, there was significant difference in birth weight $(1659+639 \mathrm{gm}$ and $3031+584)$ in the normal ratio groups. Other perinatal outcomes like APGAR score, NICU admission, no of neonatal death and IUD were significantly higher in abnormal ratio group. About $83.9 \%$ of babies who had prolonged nursery stay had abnormal ratio. Most of the babies stayed there because of Hyperbilirubenemia, Hypoglycaemia, Hypoxic isclamic enceplacopathy and hypothermia.

In the study by singh et al, $75 \%$ babies stayed at NICU for more than 10 days. One baby in the abnormal group had multiple anomalies like microcephaly and Rocker bottom foot. In the study by arias et al, 3 babies with abnormal ratio had anomalies. The birth weight of the babies at delivery were plotted in the standard birth chart and small for gestational age babies identified. In this study. 44 babies out of 90 had birth weight less than 10th percentile, $91.3 \%$ of SGA babies contributed by abnormal ratio group and only $8.3 \%$ of SGA babies contributed by normal ratio group. In study by singh et al, $94.4 \%$ of babies with abnormal ratio have less than 10th percentile babies as against $57.5 \%$ in the normal ratio group. The sensitivity, specificity, the positive predictive value and the negative predictive value of the doppler indices in predicting the adverse perinatal outcome at the cutoff values used in this study were calculated. The sensitivity of cerebroumbilical ratio to predict adverse outcome was $86 \%$ where as that for P.I of umbilical artery $(\geq 1.12)$ was $45 \%$ and for P.I. for middle cerebral artery $\leq 1.2$ was $39 \%$. The pulsatility indices of the umbilical artery and the middle cerebral artery values are useful to predict the adverse perinatal outcome. The abnormal cerebral umbilical ratio is better to predict the adverse perinatal outcome such as fetal distress, small for gestational age babies and neonatal compliations like hyperbilirubenemia in cases of IUGR along with the other antenatal surviellance. 


\section{Summary}

The doppler study indices- pulsatality indices of the umbilical artery and the middle cerebral artery and the cerebral umbilical ratio were determined in ninety antenatal women with IUGR diagnosed by clinical method, from March to October 2009. The aim of this study was to evaluate the efficacy of the cerebralumbilical ratio and the pulsatality indices of the middle cerebral and the umbilical arteries in predicting the adverse perinatal outcome in women with IUGR. The cutoff values of '1.08' for the cerebral-umbilical ratio; pulsatility indices of '1.12' for the umbilical artery and '1.2' for the middle cerebral artery were taken for this study. Women with IUGR and antenatal risk factor were found to have abnormal cerebral-umbilical ratio $(\leq$ 1.08 ; ' $\mathrm{p}$ ' value $=0.002$ ). The mean gestational age at delivery was significantly lower in women with the abnormal cerebral-umbilical ratio. More that fifty percent of women with abnormal cerebral-umbilical ratio of $\leq$ 1.08 were delivered by cesarean section.

The proportion of low birth weight of the babies were higher in women with abnormal cerebralumbilical ratio. The incidence of hyperbilirubinemia and prolonged nursery stay was found to be higher in newborns who had abnormal cerebral-umbilical ratio. 50.5\% of the women diagnosed clinically to have IUGR, delivered small for gestational age babies (birth weight $<10$ th percentile).

The sensitivity for the cerebral-umbilical ratio of $\leq 1.08$, to predict the adverse perinatal outcome in IUGR was $79.3 \%$ for pulsatility index of umbilical artery of $\geq 1.12$, it was $42.3 \%$ and for the pulsatility index of middle cerebral artery of $\leq 1.2$ was $66.6 \%$. When the cerebral-umbilical ratio is abnormal in women with IUGR, they are likely to have significant increase in perinatal risks like fetal distress, incidence of cesarean section, small for gestaitonal age babies, prolonged nursery stay and hyperbilirubinemia in the new born.

\section{Bibliography}

[1]. Fetal growth restriction: evaluation and management. In James DK, Steer PJ, Weiner CP, Gonik B. Editors - High risk pregnancy Management options - second edition. London W.B. Saunders pp. 291.

[2]. Battaglia FC, Lubchenco LO. A practical classification of newborn infants by weight and gestational age. The J Pedia 1967; 71:159 -163 .

[3]. Usher R, McLean RNF, Intrauterine growth of live-born Caucasian infants at sea level: standards obtained from measurements in 7 dimensions of infants born between 25 and 44 weeks of gestation. The J Pediatrics 1969; 74 : 901 - 910.

[4]. Mc Intire DD, Bloom SL, Casey BM et. al. Birth weight in relation to morbidity and mortality among newborn infants. N Engl. J Med. 1999; $340: 1234$ - 1238.

[5]. Rekonen A, Luotola H, Pitkanen M et. al. Measurement of intervillous and myometrial blood flow by an intravenous 133Xe method. Br. J Obstet Gynaecol 1976; 83: 723 - 728.

[6]. Stembera ZK, Hodr J, Ganz V et. al. Measurement of Umbilical cord blood flow by local thermodilution. Am J Obstet Gynecol 1964; 90: 531 - 536.

[7]. Fitz Gerald DE, Drumm JE. Non-invasive measurement of human fetal circulation using ultrasound: a new method. B M J 1977; 2: $1450-1451$.

[8]. Giles WB, Trudinger BJ, Baird PJ. Fetal umbilical artery flow velocity waveforms and placental resistance: Pathological correlation. Br. J Obstet Gynaecol 1985; 92: 31-38.

[9]. Wladimiroff JW, Wijngaard JAGW, Degani S et. al. Cerebral and umbilical arterial blood flow velocity waveforms in normal and growth retarded pregnancies. Obstet Gynecol 1987; 69: 705 - 709.

[10]. Gramellini D, Folli MC, Raboni S et. al. Cerebral-umbilical doppler ratio as a predictor of adverse perinatal outcome. Obstet Gynecol 1992; 79: 416 - 420.

[11]. Fetal growth restriction: evaluation and management. In James DK, Steer PJ, Weiner CP, Gonik B. Editors. High risk pregnancymanagement options. Second Edition. London. W.B. Saunders. pp.299.

[12]. Arias F. Accuracy of the middle - cerebral - to - umbilical artery resistance index ratio in the prediction of neonatal outcome in patients at high risk for fetal and neonatal complications. Am J Obstet Gynecol 1994; 171: 1541 - 1545.

[13]. Fetal growth disorders. In Cunningham FG, Gant NF, Leveno KJ, Gilstrap III LC, Hauth JC, Wenstrom KD. Editors. Williams Obstetrics. Twenty-first edition. London. McGraw Hill. pp.748.

[14]. Paz I, Gale R, Laor A et. al. The Cognitive outcome of full term small for

[15]. gestational age infants at late adolescence. Obstet Gynecol 1995; 85. 452-456. 15. Campbell S, Thomas A. Ultrasound measurement of the fetal head to abdomen circumference ratio in the assessment of growth retardation. Br J Obstet Gynaecol 1977; 84: 165 - 174.

[16]. Robert AB, Mitchell JM, Mc Cowan LM, et. al. Ultrasonographic measurement of liver length inthe small for gestational age fetus. Am J Obstet Gynecol 1999; 180: 634 - 638.

[17]. Fetal growth disorders. In Cunningham FG, Gant NF, Leveno KJ, Gilstrap III LC, Hauth JC, Wenstrom KD. Editors. Williams Obstetrics. Twenty-first edition. London. McGraw Hill. pp. 753.

[18]. Intrauterine growth restriction. In Creasy RK, Renik R. Editors. Maternal - Fetal Medicine - fourth edition. London. W.B. Saunders Co. pp.576.

[19]. Fetal growth restriction: Evaluation and management. In James SK, Steer PJ, Weiner CP, Gonik B. Editors. High risk pregnancy management options - second edition. London. W.B. Saunders pp. 293 - 299.

[20]. Gardosi J, Francis A. Controlled trial of fundal height measurement plotted on customised antenatal growth charts. Br J Obstet Gynaecol 1999; 106: 309 - 317.

[21]. Walraven GEL, Mkanje RJB, Van Roosmalen et. al. Single pre delivery

[22]. symphysis - fundal height meaurement as a predictor of birthweight and multiple pregnancy. Br. J Obstet Gynaecol 1995; 102: 525 -529 .

[23]. Smith GCS, Smith MFS, McNay MB et. al. The relation between fetal abdominal circumference and birthweight: findings in 3512 pregnancies. Br. J. Obstet Gynaecol. 1997; 104: 186 - 190 